\title{
Em Busca de um Framework Conceitual para Apoiar a Diversidade
}

\author{
Alberto Castro $^{1}$, Thais Castro ${ }^{1}$, Crediné Menezes ${ }^{2}$, Bruno Gadelha ${ }^{1}$, \\ Elaine Oliveira ${ }^{1}$, José Netto ${ }^{1}$, \\ ${ }^{1}$ Instituto de Computação - Universidade Federal do Amazonas (IComp/UFAM) \\ Av. Rodrigo Otávio, 6200 - 69.080-900 - Manaus - AM - Brazil \\ ${ }^{2}$ Departamento de Estudos Básicos - Faculdade de Educação - Universidade Federal do \\ Rio Grande do Sul (UFRGS) \\ Av. Paulo Gama 110, Prédio 12201 - 90.046-900 - Porto Alegre - RS - Brazil \\ \{alberto, thais, bruno, elaine, jnetto\} @icomp.ufam.edu.br, \\ credine@gmail.com
}

\begin{abstract}
In this article, we analyse the scenario of Informatics in Education from the perspective of its great challenges, focusing on questions related to the diversity of scenarios, platforms, organizations, goals, strategies, individuals and groups, arguing for a conceptual framework to reason about this complex scenario. We put in context the investigation developed by the research community, particularly the Intelligent Systems Group (GSI) linked to the Institute of Computing at the Federal University of Amazonas, Brazil, showing how these initiatives are part of an ecosystem and grouped together to tackle the diversity challenge.
\end{abstract}

Resumo. Neste artigo analisamos o cenário da Informática na Educação na perspectiva de seus grandes desafios, com foco nas questões relacionadas à diversidade de cenários, plataformas, organizações, objetivos, estratégias, pessoas e grupos envolvidos, argumentando a necessidade de um framework conceitual para refletir sobre os complexos elementos dessa questão. Situamos em tal contexto, a investigação em andamento pela comunidade de pesquisadores na área, em particular o grupo Sistemas Inteligentes (GSI) associado ao Instituto de Computação da UFAM, buscando mostrar como tais iniciativas podem ser percebidas como elementos de um ecossistema e congregadas para mitigar o desafio da diversidade.

\section{Introdução}

Vários aspectos relacionados à Informática na Educação (IE) têm motivado a pesquisa na computação e em diversas subáreas das ciências e engenharias. Um desses aspectos diz respeito à concepção, desenvolvimento e implantação de estratégias e recursos, especialmente os de natureza tecnológica, para entender e ampliar o acesso das pessoas ao conhecimento, tema alinhado a um dos Grandes Desafios científicos da computação apontados pela SBC para a década (2006-2016), aquele que trata do "acesso participativo e universal do cidadão brasileiro ao conhecimento". 
Ao longo de três décadas de investigação, uma miríade de cenários, plataformas, organizações, objetivos, estratégias, artefatos, pessoas e grupos, caracterizaram um ecossistema com características notáveis no que diz respeito à sua abrangência, complexidade e diversidade. Como consequência, as investigações em nossa comunidade frequentemente têm tratado apenas um ou outro aspecto desse ecossistema, por exemplo acerca dos serviços disponíveis nos ambientes virtuais de aprendizagem, no monitoramento das pessoas (usuários) em tais sistemas, ou ainda no uso de uma determinada tecnologia emergente, desprezando as relações entre eles e as consequentes implicações possivelmente limitantes, impostas a partir de tais soluções.

Neste artigo argumentamos pela necessidade de um framework conceitual para refletir sobre esse ecossistema, em particular no que diz respeito às implicações de nossas contribuições para as diferentes manifestações cognitivas que nele ocorrem. No sentido de elencar alguns princípios definidores de tal framework, situamos em tal contexto, a investigação em andamento pela comunidade de pesquisadores na área, em particular pelo grupo associado ao Instituto de Computação da UFAM, buscando mostrar como tais iniciativas podem ser congregadas para mitigar o desafio crescente oriundo dessa diversidade.

\section{Contexto}

Com a implementação de diversas políticas públicas buscando inserir aqueles que estão por algum motivo sem acesso à educação formal, há uma preocupação em como essa população pode ser adequadamente inserida no contexto educacional. Iniciativas como a política de cotas, regulam a participação de indivíduos em certos sistemas, mas não garantem a efetiva integração aos processos cognitivos que neles ocorrem.

Não há como desconsiderar fatores imprescindíveis que influenciam o desenvolvimento cognitivo das pessoas, como o local onde residem, suas influências culturais, as pessoas com as quais interagem em seus ambientes familiares e educativo e suas habilidades cognitivas e sociais. Nos contextos onde as pessoas convivem, desenvolvem sua cognição e constroem relações elas aprendem, de maneira formal ou informal, frequentando escolas e interagindo nas praças de suas cidades, em shoppings (nos grandes centros urbanos), na sua rua com seus vizinhos e na sua família. Em vários desses espaços de interação, elas possuem alguma forma de conectividade, mesmo que por vezes restritas a horários e locais específicos, como ocorre no interior da Amazônia.

Esse cenário de tecnologias ubíquas amplia oportunidades, embora a sociedade como um todo ainda não conseguiu incorporar, de forma sistêmica, esse fenômeno comunicacional. Cada individuo ou grupo começa a usar suas ferramentas tecnológicas, apropria-se das mesmas e segue em frente. Isso é positivo, pois se cria o hábito de conviver com as novidades, explorando e desenvolvendo novos usos. Entretanto, para que possamos aproveitar as oportunidades apresentadas, em particular no que diz respeito à educação e acesso ao conhecimento, é necessário o desenvolvimento de estudos que possibilitem um uso mais adequado dessas tecnologias.

\section{Ambientes de Aprendizagem enquanto Ecossistemas Cognitivos}

O uso do computador para apoiar a aprendizagem é um desafio que a comunidade acadêmica assumiu desde os primeiros passos desta tecnologia, cuja natureza plástica, 
que tem a capacidade de se transformar em inúmeras máquinas específicas a partir da programação em linguagens cada vez mais acessíveis, possibilita hoje o surgimento de inúmeras aplicações para diferentes usos em todas as áreas da atividade humana e notadamente no contexto educacional.

Por muito tempo, e por diferentes motivos, o mundo do trabalho, do entretenimento e da escola estiveram separados. Hoje, em função do advento das tecnologias digitais, a informação e seus usos assumiram um papel primordial dando origem ao que se tem denominado de sociedade do conhecimento. Nesta nova sociedade, o bem primordial é o conhecimento, que desde sempre, pode ser construído por cada indivíduo em diferentes contextos. Antes, as máquinas que povoavam o trabalho, o entretenimento e a escola, tinham naturezas diferentes. Na cultura digital, as máquinas compartilham um elemento primordial, os processadores programáveis e os elementos que elas produzem e consomem é a informação, que pode ser representada de forma digital.

A natureza programável dos computadores, aliada ao surgimento de linguagens de programação mais amistosas, a alta capilaridade da Internet e o surgimento de equipamentos móveis de baixo custo atraiu a curiosidade e a criatividade de jovens programadores que fazem surgir a cada dia uma grande quantidade de novos aplicativos para os usos mais diversos possíveis, em todos os âmbitos da atividade humana. Tal advento, notadamente no contexto educacional é muito bem-vindo embora, dada sua multiplicidade, apresente-nos novos desafios, como o de escolher a opção mais adequada a nossas necessidades, ou como compatibilizar as informações produzidas e consumidas por esses diferentes aplicativos.

Este aporte de diferentes ferramentas para apoiar as atividades intelectuais da sociedade do conhecimento, provoca uma transformação que assume porte de uma revolução com profundas modificações na nossa forma de agir e pensar - constatação que tem requerido um olhar mais atento da comunidade de Informática Aplicada à Educação em busca de uma fundamentação teórica que dê suporte à análise e síntese de novas iniciativas, tendo como grande desafio contemplar a diversidade em todas as suas facetas.

Não há como pensar em diversidade sem considerar o indivíduo dentro de seu próprio contexto e de seu grupo. Lévy (2010) postula a necessidade do estabelecimento de uma Ecologia Cognitiva, tendo por base uma integração, por intermédio das tecnologias digitais, entre pessoas, instituições e coisas em uma rede complexa. Tal proposta está ancorada em perspectivas como a de Baterson (1972) sobre sistemas dinâmicos e abertos, bem como as estruturas rizomáticas de Guattari (2001) e a equivalência entre humanos e não-humanos nos processos sócio-técnicos proposto por Latour (2000).

Nos dias atuais, quando se fala de aplicações educacionais, a separação entre computadores, dispositivos móveis e Internet já não importa, sendo, portanto, bastante usual a referência indissociada desses elementos. Assim, podemos postular que nosso ecossistema cognitivo, já em grande parte tem como locus a Internet. Em um recente trabalho (Smart at al, 2017) postula que partindo-se de uma perspectiva ecológica a Internet faz parte da cognição humana, pois funciona como um ambiente físico onde as atividades epistêmicas acontecem. 
É de nosso interesse adotar a abordagem ecológica como base para o estabelecimento de um framework capaz de dar suporte tanto à análise de ambientes e aplicações isoladas existentes quanto à concepção de novos ambientes para comunidades de aprendizagem e de aplicações isoladas, com respeito às suas possibilidades de atender os objetivos de uma determinada comunidade em rede.

\section{O desafio da diversidade}

Durante certo tempo o acesso aos computadores era restrito a pessoas, espaços (físicos) e situações bem específicas. Tal cenário evoluiu até o estado corrente onde a tecnologia computacional é ubíqua e parte integrante das atividades diárias da maioria de nós. Essas diferentes instâncias da tecnologia em nossas vidas apresentam uma primeira perspectiva sobre a diversidade, ainda que de natureza quase exclusivamente técnica.

A quantidade de situações, espaços e pessoas com potencial para acessar os recursos tecnológicos disponíveis ampliou-se de modo exponencial. Inúmeras combinações desses fatores (situações, espaços, pessoas, acesso) ilustram uma outra perspectiva sobre a diversidade, que necessariamente precisa agregar aspectos sociais, políticos, culturais, ambientais, dentre outros, aos técnicos.

A diversidade é um desafio para a pesquisa e a prática em Informática na Educação também porque ao mesmo tempo em que as tecnologias ubíquas e até "vestíveis" estão disponíveis para muitos, elas ainda não estão inseridas no cotidiano das escolas como uma prática para inclusão e participação do cidadão em seus próprios processos de aprendizagem.

\subsection{A experiência do GSI/UFAM}

As características de um desafio são, não raramente, percebidas mais claramente por aqueles que precisam lidar com suas implicações em ações diárias. As várias realidades vivenciadas pelos habitantes da Amazônia e a necessidade de contemplar suas peculiaridades ao mesmo tempo que se busca integrá-las a formatos rígidos inspirados em "situações-padrão", é um exemplo da percepção adquirida sobre nosso desafio.

Nesse contexto, há mais de 15 anos o GSI/UFAM tem desenvolvido pesquisa buscando contemplar a diversidade e não por acaso o tema do XV SBIE, realizado em Manaus, foi "Diversidade e Integração: Desafios para a Telemática na Educação". Algumas das dimensões como as mencionadas no início dessa seção (atores, espaços, situações e acesso) podem situar a pesquisa desenvolvida nos últimos anos pelo GSI, ilustrando parte do esforço da comunidade de IE no tema.

\footnotetext{
Atores

Atores humanos (indivíduos e grupos) e não-humanos (agentes, serviços, plataformas, etc.) são elementos centrais no ecossistema educacional e onde se tem investido em práticas participativas envolvendo os seres humanos na busca por soluções educacionais com suporte computacional e atores não-humanos como parte da solução, todos interagindo de diferentes maneiras. São exemplos dessa abordagem a utilização de agentes inteligentes (Xavier et al, 2016); com ferramentas e técnicas para melhorar a habilidade de programação na educação superior (Carvalho et al, 2016) e a elicitação e organização de conhecimento tácito cientifico (Albuquerque et al, 2016).
} 


\section{Espaços}

Espaços físicos são os lugares ou ambientes onde as interações convencionalmente aconteciam e ainda acontecem em boa parte do tempo: escolas, casas, cidades, praças, clubes, shoppings, etc. Sendo importante incorporar outros cenários, por exemplo para inserir os alunos cursando a educação formal em localidades com restrições de acesso à Internet, como descrito em (Ferreira e Castro, 2013).

Espaços virtuais são criados utilizando plataformas tecnológicas diversas. Com as oportunidades para a adoção de novas práticas pedagógicas, é cada vez mais frequente a adaptação de plataformas ou a concepção de novos paradigmas para a definição de ambientes mais flexíveis e aderentes às necessidades de indivíduos e grupos, como o paradigma MOrFEU (Santos et al, 2010).

\section{'SituAções'}

No contexto relevante para a IE, a tecnologia é um elemento constituinte das arquiteturas pedagógicas (AP) (Carvalho et al, 2005) concebidas para favorecer situações de aprendizagem e no caso do GSI, tem havido especial interesse em APs que utilizam práticas colaborativas bem como APs cuja plataforma tecnológica envolve o uso de interfaces alternativas (Lima et al, 2016).

\section{Acesso}

$\mathrm{O}$ acesso dos atores aos ecossistemas nos quais os mesmos poderiam atuar depende de um conjunto de fatores que incluem por exemplo a infraestrutura disponível no espaço físico ocupado, os aspectos sociais que orientam ou limitam as oportunidades de participação nos sistemas, ou ainda as limitações e especificidades físicas e cognitivas de cada indivíduo. No último caso mencionado, o GSI tem trabalhado na perspectiva do HCD (human-centered design), sistematizando um processo de design inclusivo (Lucke et al, 2016) e de interações multimodais (Raposo et al, 2014).

Ainda no tema "acesso", uma dimensão importante relacionada à compreensão de ecossistemas complexos é o acesso a dados provenientes dos espaços físicos e virtuais e das interações e outras situações neles ocorridas envolvendo seus atores. Através da coleta, organização, análise e reuso desses dados, o conhecimento resultante pode ser socializado e reintegrado no próprio ecossistema, como ocorre na frente de trabalho de mineração de ambientes virtuais (Almeida Neto et al, 2015).

\subsection{Colocando em perspectiva}

As dimensões apresentadas na subseção anterior possibilitam descrever parte da pesquisa desenvolvida pelo GSI/UFAM e de modo geral, pela comunidade de IE, com relação a um desafio. A análise de tal pesquisa é realizada segundo perspectivas específicas, que nos auxiliam a perceber ações fragmentadas como partes de uma rede que constitui ela mesma um "micro-ecossistema".

Em nosso caso, a definição desse "micro-ecossistema" estabeleceu conexões esquecidas ou não percebidas anteriormente, situando no desafio tratado a convergência das pesquisas de um grupo de pesquisa imerso num contexto diferenciado, embora integrado à comunidade de IE.

\subsection{Proposta para Enfrentar o Desafio}

A partir do que foi discutido aqui, o Quadro 1 apresenta um resumo com proposta de objetivos e metas/ações para tratar com asp飞ętos centrais do desafio da diversidade. 


\section{Quadro 1 - Enfrentando o Desafio da Diversidade}

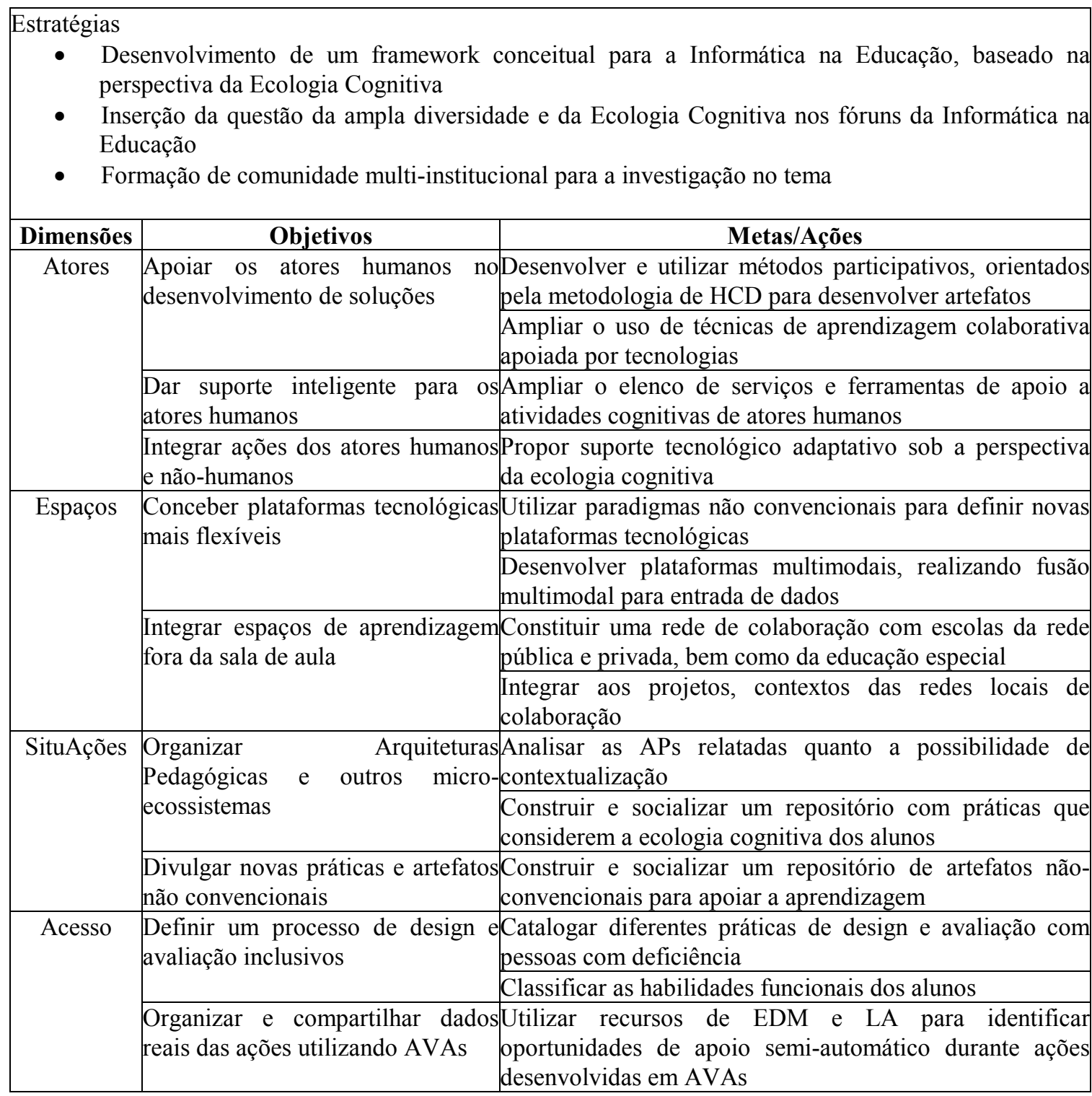

\section{Considerações Finais}

A crescente disponibilidade de equipamentos associada a uma enorme diversidade de contextos possibilita que uma grande quantidade de novos usuários chegue à rede interessados na produção de novos ambientes e aplicações. Este fenômeno é ao mesmo tempo motivo de grande entusiasmo e de grandes preocupações. O entusiasmo se dá tendo em vista que, do ponto de vista educacional, cada vez mais os jovens estão sendo protagonistas da construção de uma nova ecologia cognitiva, participando de uma atividade intelectual com grande potencial para o desenvolvimento de novas competências cognitivas. Por outro lado, essa proliferação de tecnologias sem o apoio de fundamentos e elementos estruturantes pode implicar na produção de forma fragmentada, dificultando assim as pesquisas e o desenvolvimento na área de Informática na Educação. 
$\mathrm{O}$ argumento por um framework conceitual baseado na ecologia cognitiva de cenários como o descrito acima ajudou a contextualizar a pesquisa em IE com respeito a um de seus muitos desafios segundo a perspectiva de um grupo de pesquisa típico. Os próximos passos na busca desse framework devem contemplar: maior flexibilidade nas plataformas disponíveis para indivíduos e comunidades; colaboração; suporte inteligente para a coleta, organização e uso contextualizado de dados, registro e reuso de experiências e acesso baseado em facilitadores individuais.

\section{Agradecimentos}

Parte da investigação aqui citada contou com recursos do Projeto "Framework Inteligente para Apoiar a Colaboração”, Edital FAPEAM 016/2013, Res. 035/2013.

\section{Referências}

Albuquerque, A.C.F.; Santos, J.L.C.; Castro, A. A Conceptual Framework for Integrating Scientific Tacit Knowledge. In: 2016 SAI Intelligent Systems Conference (IntelliSys), 2016, London, UK. Proceedings of 2016 SAI Intelligent Systems Conference (IntelliSys). Piscataway, NJ: IEEE Computer Society, 2016.

Almeida Neto, F.; Castro, A. Elicited and Mined Rules for Dropout Prevention in Online Courses. In: 2015 IEEE Frontiers in Education Conference (FIE). Camino Real El Paso, TX, USA, 2015.

Bateson, Gregory. Steps to an Ecology of Mind - Collected Essays in Anthropology, Psychiatry, Evolution and Epistemology. Jason Aronson Inc. 1972.

Carvalho, M.J.S.; Nevado, R.A.; Menezes, C.S. Arquiteturas Pedagógicas para Educação a Distância: Concepções e Suporte Telemático. In: XVI Simpósio Brasileiro de Informática na Educação. Juiz de Fora, 2005.

Carvalho, L.S.G.; Gadelha, B.F.; Nakamura, F.G.; Oliveira, D.B.F.; Oliveira, E.H.T. Ensino de programação para futuros não-programadores: contextualizando os exercícios com as demais disciplinas de mesmo período letivo. In: $24^{\circ}$ Workshop sobre Educação em Computação, Porto Alegre. Anais do XXXVI CSBC, 2016.

Castro, T.; Castro, A.N.; Castro, L.; Soares, M.; Gadelha, B. A Two-Level Problem Solving Practicing Approach using a Location Based App. In: XXVII Simpósio Brasileiro de Informática na Educação. Uberlândia. Anais do V CBIE, 2016.

Ferreira, R.S.; Castro, A.N. Um Esquema de Sincronismo para Ambientes Virtuais de Ensino e Aprendizagem. In: XXIV Simpósio Brasileiro de Informática na Educação. Campinas. Anais do II CBIE, 2013.

Guattari, Félix. As Três Ecologias. 11 ${ }^{\mathrm{a}}$. Ed. Trad. Maria Cristina F. Bittencourt. Papirus. Campinas, SP. 2001.

Latour, B. Ciência em ação: Como seguir cientistas e engenheiros sociedade afora. São Paulo: UNESP, 2000.

Lévy, Pierre. As Tecnologias da Inteligência - O Futuro do Pensamento na Era da Informática, $2^{\mathrm{a}}$. Ed. Trad. Caros Irineu da Costa. Editora 34. 2010. 
Lima, D.; Pereira, R.C.; Siqueira, M.P.; Castro, T.; Souza, L.J.; Freitas, J.A. MusicApp um aplicativo para apoio às crianças autistas nas aulas de musicalização. In: V Congresso Brasileiro de Informática na Educação. Uberlândia. Anais V CBIE. 2016.

Lucke, U.; Castro, T. The Process of Inclusive Design. In: IEEE 16th International Conference on Advanced Learning Technologies. Austin, MA, USA: IEEE Computer Society Conference Publishing Services (CPS), 2016. p. 446-447.

Raposo, N.; Rios, H.; Lima, D.; Gadelha, B.; Castro, T. An application of mobility aids for the visually impaired. In: Proceedings of the 13th International Conference on Mobile and Ubiquitous Multimedia - MUM '14, 2014. p. 180-189.

Santos, L.N.; Castro, A.; Menezes, C.S. MOrFEu: Criando Ambientes Virtuais Flexíveis na Web para Mediar a Colaboração. In: V Congreso Iberoamericano de Informática Educativa. Santiago, 2010. v. 1. p. 114-121.

Smart, P. R., Heersmink, R., \& Clowes, R. The Cognitive Ecology of the Internet. In: S. J. Cowley \& F. Vallée-Tourangeau (Eds.), Cognition Beyond the Brain (2nd ed.). Springer-Verlag. London, England, UK, 2017.

Xavier, N.B.; Netto, J.F.M. Optimizing the Management of Distance Courses through Multi-Agent System. In: IEEE Frontiers in Education. Erie (USA), 2016. 